\title{
Relaciones sociales y desempeño organizativo: Estudio empírico en empresas del sector hotelero
}

\author{
Leticia Rivero Almeida \\ Universidad de las Palmas de Gran Canaria \\ mapayele@hotmail.com \\ Julia Nieves Rodríguez \\ Universidad de las Palmas de Gran Canaria \\ julia.nieves@ulpgc.es
}

Recibido: 13-02-2015

Aceptado: 26-03-2016

Keywords: Social relationships; Innovation capacity; Financial performance; Market performance; Hotel establishments.

\section{ABSTRACT}

Social relationships and organizational performance: Empirical study in hotel sector firms. The purpose of this study is to contribute empirical evidence about the role played by the social relationships of members of hotel firms in the performance of these establishments. A multiple regression analysis was conducted to determine what variables could predict a hotel's capacity to improve its innovation capability and achieve financial and market results. The findings suggest that both external and internal social relationships determine the innovation capability of hotels. Moreover, external social relationships have an influence on the financial and market results. The study reveals that managers should pay special attention to the establishment of relationships with people or institutions outside the organization. In addition, the data show that the efforts made by hotel firms to create interaction and communication spaces among their employees contribute to improving the innovation capability.
Palabras clave: Relaciones sociales; Capacidad de innovación; Resultados financieros; Resultados de mercado; Establecimientos hoteleros.

\section{RESUMEN}

E1 objetivo de este estudio es aportar evidencia empírica acerca del papel que juegan las relaciones sociales de los miembros de establecimientos hoteleros sobre el desempeño de dichos establecimientos. Un análisis de regresión múltiple se realizó para determinar qué variables pueden ser predictoras de la capacidad de un hotel para mejorar su capacidad de innovación y para lograr resultados financieros y de mercado. Los hallazgos sugieren que, tanto las relaciones sociales externas como internas, determinan la capacidad de innovación de los hoteles. Asimismo, las relaciones sociales externas influyen sobre los resultados financieros y de mercado. El estudio pone de manifiesto que los gestores deben considerar con especial atención el establecimiento de relaciones con personas o instituciones externas a la organización. Asimismo, los datos muestran que los esfuerzos realizados por las empresas hoteleras para generar espacios de interacción y comunicación entre sus empleados contribuyen a mejorar la capacidad de innovación. 


\section{INTRODUCCIÓN}

La perspectiva de redes sociales surge por el reconocimiento de que numerosos individuos, equipos $u$ organizaciones están integrados en múltiples relaciones que proporcionan acceso a conocimientos, mercados, tecnologías, reputación o influencia (Inkpen y Tsang, 2005; Younget al., 2001).Está ampliamente extendida en la literatura la idea de que el establecimiento de relaciones sociales por parte de las empresas puede mejorar sus niveles de desempeño (Almeida y Phene, 2004; Inkpen y Tsang, 2005). Consecuentemente, las empresas deben tener como competencia básica la capacidad de desarrollar y gestionar relaciones con personaso instituciones ajenas a la organización,así como entre los miembros de la propia empresa (Jian y Wang, 2013).

Particularmente, la industria turística es un sector económico caracterizado por la multitud de relaciones que se establecen entre las distintas empresas e instituciones que lo conforman (Argote e Ingram, 2000). Por consiguiente, en este ámbito se crea un sistema de interrelaciones complejo, en el que confluyen diferentes tipos de organizaciones de carácter público y privado, las cuales pueden complementarse y competir a la vez (Pavlovich, 2003). La eficacia de las relaciones que se establezcan entre ellas puede influir significativamente en su capacidad para compartir conocimiento, innovar, y obtener mejores resultados; en definitiva, para seguir siendo competitivas.

Además, el turismo es una industria sometida a muchos cambios en su entorno y, consecuentemente, la capacidad de innovación constituye un requisito importante para mejorar la competitividad y asegurar la supervivencia de las empresas turísticas. En tal sentido, es esencial el desarrollo de relaciones sociales externas que permitan el acceso a conocimiento novedoso y útil para generar nuevos bienes o servicios. Asimismo, esta capacidad de innovación puede depender de la habilidad que posean los miembros de la empresa para compartir conocimientos y experiencias, esto es, de las relaciones internas que se establezcan entre susintegrantes (Subramaniam y Youndt, 2005).

Investigaciones previas han abordado el estudio de las relaciones sociales y su impacto sobre los resultados organizativos. No obstante, son muy escasos los trabajos desarrollados en la industria turística. Por consiguiente, se conoce muy poco acerca de la influencia que las relaciones sociales ejercen sobre los distintos tipos dedesempeñoorganizativo que presentan las empresas turísticas. El presente estudio se centra en analizar la influencia que ejercen las relaciones sociales en la capacidad de innovación y en los resultados, financieros y de mercado, de los establecimientos hoteleros. En concreto, se pretende evaluar, por una parte, el efecto de las relaciones sociales externas establecidas por los hoteles de cuatro y cinco estrellas situados en los puntos turísticos del sur de la isla de Gran Canaria. Asimismo, se analizará el impacto de las relaciones sociales internas que se desarrollan entre los integrantes de dichos establecimientos hoteleros. Por consiguiente, el objetivo del estudio es evaluar si las relaciones, tanto interorganizativas como intraorganizativas, favorecen el desempeño de los establecimientos hoteleros, medido en términos de capacidad de innovación, resultados financieros y resultados de mercado.

En el sur de de Gran Canaria se localiza la mayor parte de la actividad turística de la isla y, además, se trata de una zona que en los últimos años ha apostado por un turismo de calidad, donde han empezado a emerger hoteles de mayor categoria. Por consiguiente, la aplicación de esta investigación en hoteles de cuatro y cinco estrellas ubicados en esta área geográfica puede aportar conocimientos relevantes y útiles para el sector.

Para alcanzar los objetivos propuestos este estudio presenta a continuación una revisión de la literatura, seguida de la formulación de las hipótesis de investigación. En las dos secciones siguientes se exponen la metodología y los resultados de la investigación, respectivamente. Las últimas secciones se destinan a presentar las principales conclusiones e implicaciones extraídas del trabajo, asi como sus limitaciones y posibilidades de investigación futura. 


\section{MARCO CONCEPTUAL}

\subsection{La perspectiva relacional}

McEvily y Zaheer (1999) sugieren que debe revisarse una premisa básica en la teoría de recursos y capacidades (TRC), según la cual, los recursos internos son los generadores de ventajas competitivas. Para los citados autores, tales ventajas pueden tener origen externo y estar insertadas en los recursos obtenidos por las empresas debido a su pertenencia a redes sociales. Estos recursos, específicos de las organizaciones, proporcionan diferente acceso a la información, ideas y oportunidades, lo cual tiene importantes implicaciones para su capacidad competitiva (Ahuja, 2000; Dyer y Hatch, 2006; McEvily y Zaheer, 1999). En la misma línea, Molina (1999) sostiene que los límites de una empresa son insuficientes para explicar los factores que determinan la consecución de ventaja competitiva. Así, mientras la TRC señala a los recursos que posee y controla la empresa como una fuente de ventaja competitiva, la literatura refleja que la heterogeneidad resultante de los diferentes vinculos externos establecidos por las empresas es, también, un factor que determina su capacidad para alcanzar tales ventajas (Collins y Clark, 2003; McEvily y Zaheer, 1999). De este modo, los recursos críticos pueden encontrarse no solo en el interior de la organización, sino que también pueden estar insertados en recursos y rutinas interorganizativas (Dyer y Singh, 1998).

En sintesis, el enfoque relacional enfatiza que los vínculos que se establecen entre empresas pueden constituir un recurso valioso e inimitable (Capaldo, 2007; Gulatiet al., 2000). No obstante, las empresas difieren en su habilidad para identificar socios potenciales y estimar el valor de los recursos obtenidos de tal relación (Dyer y Singh, 1998). Asimismo, las oportunidades para constituir vínculos pueden estar condicionadas por los recursos en poder de una organización, debido a que el número de socios potenciales dispuestos a establecer relaciones con una empresa depende del valor añadido que esta aporte a dicha relación (Ahuja, 2000). Consecuentemente, la disponibilidad de recursos internos determina el valor de los recursos que se obtienen mediante vínculos interorganizativos. Por tanto, la posición competitiva global de una empresa es función tanto de sus recursos internos como sus los recursos interorganizativos (Kim y Oh, 2004) y, ambos, se pueden considerar inmersos en una relación simbiótica particular. Mientras el valor y las habilidades de una empresa, como integrante de una red, se relacionan con sus recursos internos, también una mayor colaboración desarrolla y fortalece dichas competencias internas (Powellet al., 1996). En otras palabras, para ser capaces de gestionar estratégicamente sus redes, las empresas necesitan un conjunto de capacidades relacionales (Capaldo, 2007), al tiempo que su participación en redes les permite desarrollar capacidades que se acumulan como resultado de un proceso de aprendizaje (Gulati, 1999). De este modo, como consecuencia de su pertenencia a redes, las empresas adquieren capacidades que aumentan su valor como posibles integrantes de una red, lo que, a su vez, puede constituir un impulso para promover nuevos acuerdos interorganizativos (Gulati, 1999; Kogut, 2000).

Por otra parte, el establecimiento de relaciones interorganizativas no es condición suficiente para acceder a recursos externos complementarios y valiosos. Para obtener recursos de red es necesario que los recursos propios sean capaces de capturar el valor creado mediante vínculos con otras empresas (Kim y Oh, 2004). En concreto, cuando se trata de acceder a recursos de conocimiento, se requiere, entre otros condicionantes, conocimiento previo relacionado que otorgue a los receptores capacidad de absorción (Cohen y Levinthal, 1989, 1990). En una relación interorganizativa, la capacidad de absorción hace referencia a que una empresa ha desarrollado la habilidad para reconocer y asimilar el conocimiento valioso de un socio en particular, lo cual está determinado por el grado en que los socios han desarrollado bases de conocimiento coincidente (Dyer y Singh, 1998; Mowery et al., 1996). Ello se explica porque los individuos pueden ser incapaces de comprender y aplicar conocimiento muy diferente al propio, aunque sea identificable y accesible (Milleret al., 2007). En tal sentido, Tsai (2009) sostiene que se necesita un determinado nivel de capacidad de absorción que favorezca el aprendizaje organizativo necesario para la 
colaboración entre empresas. De esta manera, según el citado autor, la capacidad de absorción influye en la magnitud y dirección del efecto que los diferentes tipos de colaboración tienen sobre los resultados en materia de innovación. Por su parte, Miotti y Sachwald (2003) señalan que tal capacidad ostenta una compleja influencia en la propensión a cooperar. Por un lado, la cooperación puede ser necesaria porque los recursos internos de la empresa pueden resultar insuficientes para alcanzar sus objetivos estratégicos y, por otro, la existencia de una adecuada capacidad de absorción aumenta los retornos esperados del acceso a recursos externos.

\subsection{Relaciones sociales en destinos turisticos}

La concentración de pequeñas empresas en torno a una actividad económica y en una zona geográfica determinada es un contexto que propicia las relaciones sociales y, probablemente, facilita el intercambio de conocimiento debido a la proximidad geográfica de las partes (Quintana-García y Benavides-Velasco, 2007). Por tanto, la perspectiva relacional es particularmente importante en la industria del turismo, donde diferentes tipos de organizaciones complementarias y competidoras, múltiples sectores, infraestructuras, así como un conjunto de vínculos entre sector público y privado, forman un complejo sistema de interrelaciones para crear una estructura de oferta diversa y muy fragmentada (Pavlovich, 2003; Sorensen, 2007; Tinsley y Lynch, 2001).

Desde la perspectiva del visitante, las diferentes actividades que ofrece un destino turístico conforman una experiencia global que va a determinar su valoración de la zona (Cooper, 2006). Esto sugiere que, a pesar de la fragmentación de la oferta, es necesario que se produzca un intercambio de conocimiento efectivo en los destinos, aspecto no exento de dificultades por la existencia de organizaciones que compiten y comparten a la vez, y a las que es necesario convencer de que ésta cooperación aumentará su competitividad (Cooper, 2006; Scott y Laws, 2006). Novelli et al.(2006) señalan que debido a la naturaleza diversa de las pequeñas y medianas empresas implicadas, el desarrollo de redes en turismo no puede contemplarse como un proceso sencillo y espontáneo. En la misma línea, Hjalager (2002) sostiene que existe poca confianza mutua entre las empresas de turismo que se consideran, a menudo, como competidoras. Aún cuando muchos destinos son fuertemente dependientes del turismo y muchas empresas no podrían subsistir sin la presencia del resto, la colaboración entre ellas suele requerir la intermediación de otros organismos(Hjalager, 2002; Novelliet al., 2006).

No obstante, el intercambio de conocimiento es esencial en los destinos turísticos porque permite a un grupo de pequeñas y medianas empresas competir globalmente mediante la cooperación a nivel local (Cooper, 2006; Novelliet al., 2006). Para Scottet al. (2008), es indispensable tal colaboración para promover la competitividad de los destinos turísticos puesto que éstos están conformados por grupos de proveedores independientes y débilmente articulados, pero vinculados entre sí por participar en la prestación del servicio como un todo. Novelliet al. (2006) señalan que las redes en turismo han aportado a las empresas beneficios tales como flexibilidad, intercambio de información de mercado valiosa, capacidad de innovación, oportunidad para entrar en otras redes, desarrollo de recursos, y transferencia de conocimiento. Además, mediante actividades de cooperación e información compartida, los potenciales innovadores pueden reducir costes, riesgos e incertidumbre asociados a los procesos de innovación (Ordanini y Maglio, 2009).

También, Wang y Fesenmaier (2007) sostienen que los acuerdos de colaboración en turismo son esenciales para la competencia de un destino, al proporcionar productos y servicios complementarios y ofrecer una experiencia holística al viajero. Estos autores analizan las alianzas establecidas por organizaciones de turismo para participar en la comercialización y promoción de un destino de forma colectiva y destacan que el aprendizaje es una de las consecuencias más importantes de dicha colaboración. Las partes directamente afectadas están interesadas en aportar su conocimiento, experiencia y otras capacidades a los esfuerzos de comercialización colaborativa lo cual, a su vez, permite 
cambios, mejoras e innovación a través de los procesos de aprendizaje. Otra consecuencia importante de la colaboración es que permite disponer de recursos tales como información, ideas, oportunidades de negocio, poder e influencia, apoyo emocional, confianza y espíritu de cooperación.

Por su parte, Saxena (2005) se centra en la cooperación en marketing de destinos. Para esta autora, la necesidad de un enfoque cooperativo en los destinos turísticos surge como consecuencia de un cambio en las estrategias competitivas influenciadas por la volatilidad y la sensibilidad existente en la industria del turismo. La transformación radical de la industria del turismo provocada por la importancia otorgada a la calidad, el rápido desarrollo de las tecnologías de la información y la madurez de los consumidores, requiere que los actores clave de la industria del turismo se planteen formas sensatas de combinar sus recursos y actividades.

Pavlovich (2003) utiliza la teoría de redes para ilustrar como la escasez de vínculos relacionales dentro de un destino, contribuyen a limitar los recursos y los flujos de información. Por el contrario, permitir la proliferación de vínculos relacionales contribuye a un intensivo intercambio de información que favorece la creación de conocimiento. De este modo, las estructuras de la red poseen recursos basados en conocimiento que pertenecen al colectivo interdependiente, sin que ninguna organización ostente el control o la propiedad de estos activos.

\subsection{Relaciones intraorganizativas}

Los diferentes tipos de empleados dentro de una empresa cuentan con conocimiento inestimable relativo a asuntos internos y externos de la compañía, que les proporciona capacidad para realizar propuestas acorde a su capacidad tecnológica, de producción o de comercialización (Avlonitis et al.,1994). De ahí, que parte de la literatura resalte que debe ser la base de conocimientos presente en la empresa la que determine la manera en que ésta configura su estructura organizativa y que ésta debe ser apropiada para facilitar flujos de comunicación efectivos entre los diferentes individuos, grupos o departamentos (Kumar et al., 2008). Grant (1997) sostiene que, si una parte importante del conocimiento es tácito $\mathrm{y}$, por tanto, difícil de transferir, la jerarquía no es eficaz como mecanismo para la integración del conocimiento, dado que un directivo no puede integrar eficientemente el conocimiento de sus subordinados.

La interacción social permite el intercambio de ideas y enriquece la información entre los miembros de un grupo de trabajo, colaborando en un proceso interactivo de fortalecimiento de conocimiento que les permite no sólo aprovechar más eficientemente el conocimiento existente sino, además, perfeccionarlo (Subramaniam y Youndt, 2005). De ahí la importancia de una cultura organizativa que fomente las relaciones entre grupos implicados en procesos críticos de la empresa.Nonaka y Takeuchi (1995) resaltan como característica de las empresas japonesas el que ningún departamento o grupo tiene la responsabilidad exclusiva de crear conocimiento sino que este proceso es resultado de una interacción dinámica entre todos los empleados de la empresa.Asimismo, Bartmess y Cerny (1993) señalan que el desarrollo y mantenimiento de capacidades esenciales exige una comunicación informal, constante y frecuente que debe reemplazar a los contactos formales y periódicos. Por su parte, Starbuck (1992) sostiene que el trabajo en equipo facilita la divulgación de la información y fomenta la lealtad al grupo. Tsai (2001) destaca que cuando el conocimiento se transfiere entre distintos departamentos de la empresa proporciona oportunidades para el aprendizaje mutuo y la cooperación, los cuales favorecen el desempeño organizativo

\section{HIPÓTESIS DE INVESTIGACIÓN}

\subsection{Relaciones sociales externas y desempeño organizativo}

Jian y Wang (2013) sostienen que el desempeño organizativo depende en gran medida de aquellos con los que se interactúa. Por consiguiente, la capacidad de una 
empresa para establecerrelaciones con proveedores, clientes y otras organizaciones, y para gestionar eficazmente estas interacciones, es una competencia básica de las empresas que tiene una relación directa con su ventaja competitiva y su desempeño. Los participantes en procesos de interacción social pueden, no solo hacer suyo el conocimiento que ya poseen otros, sino, además, generar nuevo o emergente conocimiento que no posee ningún miembro con anterioridad, puesto que surge de la propia interacción (Argote, 1999). Ello se explica porque las relaciones que se establecen pueden conducir a nuevas interpretaciones que derivan en otro nivel de conocimiento y, es ésta evolución del conocimiento, la que da origen a las innovaciones (Carneiro, 2000). Por el contrario, cuando el conocimiento no se comparte, los recursos cognitivos disponibles estarán infrautilizados y no se desarrollará conocimiento emergente, el cual es particularmente importante cuando se participa en tareas que implican creatividad e innovación (Argote, 1999). Por consiguiente, el conocimiento necesario para desarrollar innovaciones no solo está disponible dentro de la empresa, sino que es posible acceder a él estableciendo relaciones externas (Ordanini y Maglio, 2009; Powell et al., 1996). De este modo, dado que la innovación se basa en ideas de diferente origen, las empresas pueden ampliar sus posibilidades de innovar accediendo a un mayor número de fuentes de conocimiento (Leiponen y Helfat, 2010). En tal sentido, Laursen y Salter (2006) afirman que una búsqueda amplia y profunda de conocimiento e información a través de distintos canales externos proporciona ideas y recursos que ayudan a las empresas a obtener y explotar oportunidades de innovación.

Ooi et al.(2014) destacan la importancia de las relaciones sociales de los miembros del equipo directivo en empresas del sector turístico para lograr mejoras en la calidad de los servicios que se ofrecen. El turista no consume el producto de un solo proveedor, sino un conjunto de servicios de diferentes proveedores que participan en la creación de una "experiencia" de turismo, por lo que la evaluación de los clientes depende de la percepción acumulada de múltiples servicios (Weiermair (2006). Así, por ejemplo, los vínculos establecidos entre las empresas hoteleras y las agencias de viajes pueden facilitar la colaboración y mejorar, de este modo, la experiencia de viaje de los turistas. En esta misma linea, Carpenter y Westphal (2001) sostienen que, al tener las empresas turísticas que revisar sus estrategias con frecuencia para adaptarse a los entornos inestables, es necesario una mayor diversidad de relaciones externas, las cuales pueden determinar los resultados de las empresas. También, Lee et al.(2001) señalan que los contactos externos realizan un papel muy importante en la identificación de oportunidades de emprendimiento. Por su parte, Sarkar et al.(2001) sostienen que la pertenencia a un red externa supone una fuente potencial de creación de valor, especialmente en lo que se refiere a obtener fuentes de innovación, y por tanto una mejora del rendimiento organizativo. Para estos autores las empresas con propensión a establecer relaciones externas tienden a lograr mayores resultados, los cuales se traducen en el crecimiento de las ventas, la cuota de mercado, y el desarrollo de nuevos productos y mercados.

Atendiendo a los anteriores planteamientos, consideramos que el acceso a información y conocimiento novedoso situado fuera de los limites organizativos es un elemento esencial para mejorar la capacidad de innovación de los establecimientos hoteleros. Asimismo, proponemos que tales relaciones pueden mejorar los niveles de desempeño, medidos en términos de resultados financieros y de mercado. Por consiguiente, establecemos las siguientes hipótesis de investigación:

Hipótesis 1a: Cuanto mayor sea el nivel de relaciones sociales externas de los miembros de los establecimientos hoteleros, mayor será la capacidad de innovación de dichos establecimientos.

Hipótesis 1b: Cuanto mayor sea el nivel de relaciones sociales externas de los miembros de los establecimientos hoteleros, mayores serán los resultados financieros de dichos establecimientos. 
Hipótesis 1c: Cuanto mayor sea el nivel de relaciones sociales externas de los miembros de los establecimientos hoteleros, mayores serán los resultados de mercado de dichos establecimientos.

\subsection{Relaciones sociales internas y desempeño organizativo}

Las relaciones sociales internas permiten a los miembros de una empresa obtener información acerca de la existencia, localización y relevancia del conocimiento contenido en otros departamentos o unidades organizativas (Hansen 2002). Ordanini y Parasurman (2011) destacan la importancia de establecer mecanismos formales e informales adecuados para captar y compartir el conocimiento de los empleados de primera línea en las organizaciones de servicios. Los citados autores sostienen que éstos empleados contribuyen al fomento de la innovación debido a su proximidad y frecuente interacción con los clientes y al conocimiento que obtienen mediante su experiencia acerca de cómo las cosas se podrian o deberian hacer para mejorar el servicio al cliente. Por consiguiente, los directivos deben reconocer que las habilidades y conocimiento de los empleados permiten sugerencias creativas, nuevas propuestas y actividades de investigación que posibilitan la generación de innovaciones (Carneiro, 2000).

Paralelamente, Subramaniam y Youndt (2005) sostienen que la complejidad inherente a muchas innovaciones requiere la puesta en común e integración de los conocimientos individuales y, por tanto, es indispensable que las empresas proporcionen los canales necesarios para que los trabajadores estén conectados y compartan experiencias. Ellos demuestran que la comunicación, la difusión fluida de información así como el intercambio y asimilación de conocimientos, son elementos fundamentales que determinan la capacidad de innovación de las empresas. También Leenders et al.(2003) afirman que mejorar el nivel de interacción entre los empleados aumenta la probabilidad de intercambiar ideas y esto conduce a más y mejores ideas nuevas. En la misma línea, Van de Ven (1986) destacan que, si bien la invención o la concepción de ideas innovadoras puede ser una actividad individual, la innovación representa un logro colectivo.

También, la interacción entre empleados es importante porque fomenta el desarrollo de opiniones y valores compartidos que facilitan la formación de conductas de grupo, las cuales pueden beneficiar a al conjunto de la organización (Liu, 2013; Perry-Smith, 2006). Así, las relaciones entre los distintos empleados y departamentos se considera un antecedente del desempeño organizativo medido en términos de mejora de la calidad de las relaciones con los clientes, de la coordinación en funciones de aprovisionamiento y venta así como de marketing interno (Mitrega, 2012).

Consecuentemente, gran parte de la literatura resalta la importancia de una cultura organizativa que fomente las relaciones tanto entre los distintos departamentos de la empresa, como entre los diferentes individuos que los integran. Por tanto, en este estudio formulamos las siguientes hipótesis de investigación:

Hipótesis 2a: Cuanto mayor sea el nivel de relaciones sociales internas entre los miembros de los establecimientos hoteleros, mayor será la capacidad de innovación de dichos establecimientos.

Hipótesis 2b: Cuanto mayor sea el nivel de relaciones sociales internas entre los miembros de los establecimientos hoteleros, mayores serán los resultados financieros de dichos establecimientos.

Hipótesis 2c: Cuanto mayor sea el nivel de relaciones sociales internas entre los miembros de los establecimientos hoteleros, mayores serán los resultados de mercado de dichos establecimientos. 


\section{METODOLOGÍA DE LA INVESTIGACIÓN}

\subsection{Población, recogida de información y muestra}

En este trabajo de investigación la población objeto de estudio está constituido por los establecimientos hoteleros de cuatro y cinco estrellas situados en los municipios de San Bartolomé de Tirajana y Mogán. La información relativa a la población se obtuvo a partir de los datos del Patronato de turismo de Gran Canaria. Según los registros del Patronato, dichos municipios cuentan con un total de 52 establecimientos hoteleros que ostenten la categoría de cuatro o cinco estrellas. Se decidió someter a estudio a todos los establecimientos que constituían la población, por lo que se remitió el cuestionario a los 52 hoteles. El método de investigación elegido fue la encuesta instrumentada a través de un cuestionario y la recogida de datos se realizó entre febrero y mayo del año 2015. Durante este periodo se contactó hasta en seis ocasiones con los establecimientos hoteleros que constituian la población, tanto personalmente como vía e-mail. El proceso de recogida de datos finalizó con la recepción de un total de 34 cuestionarios cumplimentados, lo cual representa una tasa de respuesta del $65,38 \%$.

\subsection{Variables de medida}

La medición de las variables se realizó mediante escala Likert de 7 puntos, donde el valor 1 indicaba total desacuerdo del informante con las afirmaciones y el valor 7 que estaba totalmente de acuerdo con las mismas. Para medir las relaciones sociales externas se adaptó la escala utilizada por Molina-Morales y Martínez-Fernández (2009). Las relaciones sociales internas se midieron sobre la base de la escala propuesta por Cabello-Medina et al. (2011). La escala para medir la capacidad de innovación se basó en la desarrollada por Rhee et al.(2010). Finalmente, las escalas que miden los resultados, financieros y de mercado, se adaptaron del trabajo de Grissemann et al.(2013).

En este estudio se controló estadísticamente el efecto del tamaño y la antigüedad de la empresasobre la capacidad de innovación, los resultados financieros y los resultados de mercado. El uso de variables de control permite obtener estimaciones más precisas de las relaciones analizadas porque se considera que dichas variables no están vinculadas a las hipótesis que se plantean pero pueden producir distorsiones en los resultados obtenidos. La literatura en general presenta resultados dispares respecto a la relación entre el tamaño de la empresa y su capacidad de innovación. No obstante, los trabajos realizados en el sector turístico son casi unánimes presentando una asociación positiva entre dichas variables (Martínez-Ros y Orfila-Sintes, 2009; Pikkemaat y Peters, 2005). Asimismo, dado que las empresas más grandes cuentan con ventajas, tales como economías de escala, mayor poder de negociación y más capacidad financiera, investigaciones previas señalan que el tamaño de la empresa puede estar relacionado con los resultados organizativos (Amara et al., 2008; Avermaete et al.,2003; Hsu y Sabherwal, 2012).

Por su parte, la antigüedad de la empresa se ha relacionado con la capacidad de innovación debido a que las empresas establecidas cuentan con mayor capacidad técnica y financiera para adaptarse a los cambios (Amara et al.,2008; Jiménez-Jiménez and SanzValle, 2010). Finalmente, respecto a la relación entre antigüedad de la empresa y resultados organizativos, los investigadores han encontrado tanto relaciones negativas (Chadwick et al., 2014), como positivas (Tajeddini y Trueman, 2012; Wang y Bansal, 2012), y no significativas (Tajeddini, 2010). El tamaño y la antigüedad se midieron mediante la transformación a logaritmo natural del número de empleados y de los años en funcionamiento de los establecimientos hoteleros, respectivamente.

\section{ANÁLISIS Y RESULTADOS}

El análisis de los datos incluyó análisis factorial exploratorio(AFE) y análisis de regresión múltiple,usando el paquete estadístico para ciencias sociales SPSS versión 22.La fiabilidad de las escalas utilizadas se evaluó a partir del coeficiente alfa de Cronbach. ElAFE confirmó la existencia de una sola dimensión en las variables utilizadas, a excepción de la 
escala que mide las relaciones sociales internas. En el análisis realizado sobre esta variable, las cargas factoriales mostraron la existencia de dos dimensiones que presentan racionalidad teórica. El primer factor incluye específicamente relaciones en las que están implicados los directivos. El segundo factor engloba relaciones internas que afectan a los miembros del hotel en general. Las cargas factoriales son superiores a 0,7 en los items de todas las escalas, superando, por tanto, el umbral de 0,50 recomendado. Asimismo, el alfa de Cronbach presenta valores aceptables en todas las variables (superiores a 0,86), a excepción del estadístico que mide la fiabilidad de las relaciones sociales internas entre los miembros del hotel en general, que se sitúa en 0,645 lo que indica un nivel de fiabilidad algo débil. Los datos detallados se muestran en el anexo 1.

Para contrastar las hipótesis formuladas se realizaron análisis de regresión múltiple. Este análisis tiene por objetivo predecir los cambios en la variable dependiente en respuesta a cambios en las variables independientes. El coeficiente de determinación $\left(R^{2}\right)$ indica qué porcentaje de la variación en la variable dependiente se explica por las variables independientes. El coeficiente de regresión (Beta) denota el cambio estimado en la variable dependiente por un cambio unitario de la variable independiente. Finalmente, la prueba $t$, y su nivel crítico $(p)$ sirve para contrastar la hipótesis nula de que un coeficiente de regresión toma el valor cero. Para descartar los problemas de multicolinealidad, que pueden reducir el poder predictivo de las variables independientes, se usó el nivel de tolerancia, el cual adopta valor 1 cuando una variable no comparte ningún porcentaje de su varianza con el resto de variables independientes.

El análisis de regresión sobre las variables dependientes se realizó incorporando las tres variables independientes que resultaron del análisis factorial exploratorio, esto es, las relaciones externas, las relaciones internas del equipo directivo y las relaciones internas que implican al resto de empleados, además de las variables de control (tamaño y antigüedad de la empresa). Se utilizó la técnica denominada de pasos sucesivos. Se trata de un método de construcción de la ecuación de regresión lineal múltiple que selecciona las variables paso a paso. En cada etapa se introduce la variable independiente que no se encuentre ya en la ecuación y que tenga la probabilidad de $F$ más pequeña, si esa probabilidad es suficientemente pequeña. Las variables ya introducidas en la ecuación de regresión se eliminan de ella si su probabilidad de $F$ llega a ser suficientemente grande. El método termina cuando ya no hay más variables candidatas a ser incluidas o eliminadas. La tabla 1 muestra los resultados del análisis para cada variable dependiente. Los niveles de tolerancia oscilan entre 0,60 y 0,90 , indicando que no existen problemas de multicolinealidad. Asimismo, el $p$-valor asociado al estadístico $F$ indica que existe un efecto significativo de las variables independientes sobre las tres variables dependientes analizadas.

Como puede observarse en la tabla 1, las relaciones sociales externas tienen un peso significativo en lastres ecuaciones de regresión realizadas. Estos resultados nos llevan a aceptar las hipótesis $\mathrm{H} 1 \mathrm{a}, \mathrm{H} 1 \mathrm{~b}$ y H1c. Por su parte, las dos dimensiones de las relaciones sociales internas analizadas se relacionan positivamente con la capacidad de innovación, por lo que se acepta la H2a. Los resultados muestran que dichas relaciones internas influyen más significativamente sobre la capacidad de innovación que las relaciones externas. No existe, sin embargo, evidencia empírica para los vínculos propuestos entre las relaciones sociales internas y los resultados financieros y de mercado. Finalmente, los datos reflejan que las variables de control contempladas no presentan efectos significativos sobre las variables dependientes. 
Rivero Almeida, L. y Nieves Rodríguez, L. (2016): "Relaciones sociales y desempeño organizativo: Estudio empírico en empresas del sector hotelero"

Tabla 1. Análisis de regresión múltiple

\begin{tabular}{|c|c|c|c|c|c|c|c|}
\hline \multirow{3}{*}{ Variables independientes } & \multirow[b]{3}{*}{ Tolerancia } & \multicolumn{6}{|c|}{$\begin{array}{l}\text { Variables } \\
\text { dependientes }\end{array}$} \\
\hline & & \multicolumn{2}{|c|}{$\begin{array}{l}\text { Capacidad de } \\
\text { innovación }\end{array}$} & \multicolumn{2}{|c|}{$\begin{array}{l}\text { Resultados } \\
\text { financieros }\end{array}$} & \multicolumn{2}{|c|}{$\begin{array}{l}\text { Resultados de } \\
\text { mercado }\end{array}$} \\
\hline & & Beta & t-value & Beta & $t$-value & Beta & $t$-value \\
\hline Relaciones sociales externas & 0,711 & 0,303 & $2,230^{*}$ & 0,402 & $2,487^{*}$ & 0,458 & $2,911^{* *}$ \\
\hline Relaciones sociales internas (directivos) & 0,731 & 0,468 & $3,408^{* *}$ & - & - & & \\
\hline $\begin{array}{l}\text { Relaciones sociales internas (empleados) } \\
\text { Variables de control }\end{array}$ & 0,602 & 0,404 & $2,945^{* *}$ & - & - & & \\
\hline Tamaño de la empresa & 0,901 & - & - & - & - & & \\
\hline Antigüedad de la empresa & 0,782 & - & - & - & - & & \\
\hline$R^{2}$ & & \multicolumn{2}{|c|}{0,585} & \multicolumn{2}{|c|}{0,162} & \multicolumn{2}{|c|}{0,209} \\
\hline$R^{2}$ Ajustado & & \multicolumn{2}{|c|}{0,543} & \multicolumn{2}{|c|}{0,136} & \multicolumn{2}{|c|}{0,185} \\
\hline Cambio en $F$ & & \multicolumn{2}{|c|}{$4,974^{*}$} & \multicolumn{2}{|c|}{$6,184^{*}$} & \multicolumn{2}{|c|}{$8,472^{* *}$} \\
\hline
\end{tabular}

Tamaño de la muestra: 34 


\section{DISCUSIÓN Y CONCLUSIONES}

El objetivo de este trabajo ha sido evaluar la influencia quelas relaciones sociales externas e internas en las que se integran losmiembros de los establecimientos hoteleros de cuatro y cinco estrellas, situados en el sur de la isla de gran canaria, ejercen sobre la capacidad de innovación, los resultados financieros y losresultados demercado de dichos establecimientos. Aunque los recursos relacionales han sido objeto de amplio tratamiento por parte de los investigadores en los últimos años, pocos trabajos han evaluado su efecto en el sector el turístico, considerado crítico para la economía de muchos países. Los resultados muestran que también en este campo de la actividad económica los recursos intangibles a los que puede accederse participando en redes sociales deben contemplarse cuando se trata de explicar la capacidad innovadora y los resultados de los establecimientos hoteleros.

Se evidenció que las relaciones sociales externas influyen en la capacidad de innovación y en los resultados, tanto financieros como de mercado de los establecimientos hoteleros.La literatura manifiesta consenso respecto a que las actividades de interacción social constituyen fuente de innovaciones porque favorecen los procesos de transferencia, intercambio y combinación de conocimiento (Inkpeny Tsang, 2005; Kang et al., 2007; NahapietyGhoshal, 1998). Asimismo, los datos son consistentes con los argumentos de Carpenter y Westphal (2001), para quienes una mayor diversidad en las relaciones externas de las empresas turísticas representa un modo de adaptación a los entornos inestables que mejora los resultados organizativos. En línea con tales argumentos, los resultados sugieren que la exposición a una amplia variedad de información mejora el nivel de información y conocimiento de los miembros de los hoteles y, consecuentemente, su capacidad de innovación y su habilidad para adoptar las medidas adecuadas que favorezcan el logro de mejores resultados organizativos.

Atendiendo a los resultados de los análisis estadísticos realizados, se evaluó las relaciones sociales internas considerando dos dimensiones, por un lado las relaciones que afectan a los miembros del equipo directico y, por otro, las relaciones existentes entre los empleados en general. Los datos muestran que ambos tipos de relaciones ejercen una influencia positiva y significativa en la capacidad de innovación de la empresa pero no se encontró relación significativa con los resultados financieros ni con los resultados de mercado. Este resultado sugiere que los esfuerzos realizados por los establecimientos hoteleros para que se produzca una comunicación frecuente entre sus miembros favorecen el intercambio y la combinación de conocimiento, lo que contribuye a mejorar la capacidad de innovación de los hoteles. No obstante, se necesita investigación adicional para contrastar el vínculo entre relaciones internas y procesos de intercambio y combinación de conocimiento. Esta investigación solo muestra que dichas relaciones internas constituyen un antecedente de la capacidad de innovación de los hoteles.

Desde un punto de vista teórico, esta investigación ha puesto de manifiesto que, en el ámbito de las empresas turísticas, los recursos valiosos no solo se encuentran dentro de los limites organizativos, sino que pueden estar insertados en su red de relaciones externas. Además, los resultados de esta investigación contribuyen a ampliar la información que precisan los gestores de las empresas hoteleras para mejorar la toma de decisiones. Actualmente, las empresas de alojamiento turístico desempeñan su actividad en un entorno altamente competitivo y, en tales circunstancias, las posiciones ventajosas en el mercado pueden perderse con relativa facilidad. Se precisa, por tanto, emprender acciones para hacer frente a las condiciones cambiantes, intentando mantener o mejorar las ventajas competitivas mediante la capacidad de innovación y la consecución de mejores resultados organizativos. En tal sentido, este estudio pone de manifiesto que los gestores deben considerar con especial atención el establecimiento de relaciones con personas o instituciones ajenas al establecimiento hotelero por el efecto que ejercen sobre el desempeño de los establecimientos hoteleros.Asimismo, las relaciones intraorganizativas parecen constituir un elemento clave para mejorar la capacidad de innovación de las empresas hoteleras. 


\section{LIMITACIONES E INVESTIGACIÓN FUTURA}

Este estudio presenta limitaciones que sugieren investigación futura. Una limitación surge del uso de la técnica del informante clave, dada la imposibilidad de contar con un número suficiente de múltiples informantes. Se intentó mitigar el posible sesgo perceptual asegurándonos, en alguna medida, la fiabilidad de las respuestas. Por esta razón, los informantes de la muestra obtenida cuentan con amplia experiencia profesional y ocupan posiciones de responsabilidad, lo que les permite una visión global de la organización. Otra limitación se relaciona con la posibilidad de generalizar las conclusiones obtenidas. Los resultados alcanzados solo pueden extrapolarse al ámbito de la población objeto de estudio, esto es, a los establecimientos hoteleros de cuatro y cinco estrellas situados en el sur de la isla de Gran Canaria. Por tanto, podria ser interesante aplicar el modelo en una muestra más amplia de establecimientos, así como realizar posibles estudios comparativos entre diferentes destinos turísticos. Asimismo, podría ampliarse el estudio analizandoel posible efecto mediador que otras variables, tales como los procesos de intercambio y combinación del conocimiento, ejercen en el vínculo relaciones sociales-desempeño organizativo.

Contemplar el estudio de las relaciones sociales como recursos independientes representa otra limitación del trabajo. Ambos tipos de redes pueden comportarse como recursos complementarios o sustitutivos. Ello implica que el valor de cada tipo de relación puede aumentar o disminuir ante la presencia del otro. Constituye, por tanto, otra posible via de investigación la incorporación de tales efectos sistémicos que pongan de manifiesto si el conjunto es mayor, o menor, que la suma de las partes. 
Anexo 1. Análisis factorial exploratorio Items

Cargas

factoriales

Relaciones sociales externas. Alfa de Cronbach: 0,860; Varianza explicada: 70,796

RE_1 Los directivos de este hotel dedican una considerable cantidad de tiempo a compartir ideas e impresiones con personas de otras organizaciones (competidores, consultores, organismos públicos, etc.).

RE_2 Los directivos de este hotel dedican una considerable cantidad de tiempo a participar en eventos sociales relacionados con su trabajo.

RE_3 Se producen frecuentes contactos entre los directivos de este hotel y el resto de las empresas instaladas en las zonas.

RE_4 Los miembros de este hotel mantienen contactos frecuentes con los clientes, agencias de viajes, tour operadores, proveedores, etc.

Relaciones sociales internas. Alfa de Cronbach, factor 1: 0,860; Alfa de Cronbach, factor 2: 0,635; Varianza total explicada:75,472

RI_1 Existe una comunicación frecuente entre los integrantes del equipo directivo de este hotel.

RI_2 Existe una comunicación frecuente entre los directivos y los responsables de los distintos departamentos del hotel.

RI_3 Los empleados pertenecientes a distintos departamentos establecen con frecuencia contactos de trabajo para compartir ideas e impresiones.

RI_4 Existe una comunicación fluida y fácil entre los miembros de un mismo departamento.

RI_5 Convocamos con frecuencia reuniones para que los miembros de este hotel compartan ideas e impresiones.

Capacidad de innovación. Alfa de Cronbach: 0,913; Varianza explicada: 79,596

CI_1 Los directivos de este hotel buscan activamente ideas innovadoras.

CI_2 En este hotel, la innovación se percibe como constructiva y es fácilmente aceptada.

CI_3 En este hotel se anima a los empleados a aportar nuevas ideas.

CI_4 En este hotel se fomenta la innovación.

Resultados financieros. Alfa de Cronbach: 0,984; Varianza explicada: 95,314

RF_1 En los dos últimos años este hotel ha sido rentable.

RF_2 En los dos últimos años este hotel ha logado los objetivos de beneficios. ,983

RF_3 En los dos últimos años este hotel ha logrado los objetivos de ventas. ,979

RF_4 En los dos últimos años este hotel ha logrado los objetivos de cuota de mercado.

Resultados de mercado. Alfa de Cronbach: 0,893; Varianza explicada:77,285

RM_1 En los dos últimos años hemos mejorado la lealtad de los clientes existentes.

RM_2 En los dos últimos años hemos atraído un número significativo de nuevos clientes.

RM_3 En los dos últimos años ha aumentado la satisfacción del cliente.

RM_4 En los dos últimos años se ha fortalecido la imagen percibida de este hotel. 


\section{BIBLIOGRAFÍA}

Almeida, P. y Phene, A. (2004):"Subsidiaries and knowledge creation: The influence of the MNC and host country on innovation",Strategic Management Journal, 25(8-9): 847-864.

Amara, N., Landry, R., Becheikhb, N. y Ouimet, M. (2008):"Learning and novelty of innovation in establishedmanufacturingSMEs", Technovation, 28(8): 450-463.

Argote, L. (1999): Organizationallearning: Creating, retaining, and transferringknowledge, New York, KluwerAcademic Publisher.

Argote, L. e Ingram, P. (2000):"Knowledge transfer: A basis for competitive advantage in firms",Organizational Behavior and Human Decision Processes,82(1): 150-169.

Ahuja, G. (2000):"Theduality of collaboration: Inducements and opportunities in theformation of inter-firmlinkages",Strategic Management Journal, 21(3): 317-343.

Avermaete, T., Viaene, J., Morgan, E. J. y Crawford, N. (2003):"Determinants of innovation in smallfoodfirms",EuropeanJournal of Innovation Management, 6(1): 8-17.

Avlonitis, G., Kouremenos, A. y Tzokas, N. (1993): "Assesing the innovativeness of organizations and its antecedents: Project Innovstrat",European Journal of Marketing,28(11): 5-28.

Bartmess, A. y Cerny, K.(1993):"Building competitive advantage through a global network of capabilities",California Management Review, 35(2): 78-104.

Cabello-Medina, C., López-Cabrales, A. y Valle-Cabrera, R. (2011):"Leveragingtheinnovative performance of human capital through HRM and social capital in Spanishfirms",International Journal of Human Resource Management,22(4): 807-828.

Capaldo, A. (2007):"Network structure and innovation: theleveraging of a dual network as a distinctiverelationalcapability",Strategic Management Journal, 28(6): 585-608.

Carneiro, A. (2000): "Howdoesknowledgemanagementinfluenceinnovation and competitiveness?"Journal of Knowledge Management, 4(2): 87-98.

Carpenter, M. A. y Westphal, J. D. (2001):"The strategic context of external network ties: examining the impact of director appointments on board involvement strategic decision making",Academy of Management Journal, 4(4): 639-660.

Chadwick, C., Super, J. F. y Kwon, K. (2014):"Resourceorchestration in practice: CEO emphasison SHRM, commitment-based HR systems, and firm performance",Strategic Management Journal, 36(3): 360-376.

Cohen, W. y Levinthal, D. (1989):"Innovation andlearning: Thetwo faces of $R \& D^{\prime \prime}$,TheEconomicJournal, 99: 569-596.

Cohen, W. y Levinthal, D. (1990):"Absorptivecapacity: A new perspectiveonlearning and innovation",AdministrativeScienceQuarterly, 35(1): 128-152.

Collins, C. J. y Clark, K. D. (2003):"Strategic human resourcepractices, top managementteam social networks, and firm performance: The role of human resourcepractices in creatingorganizationalcompetitiveadvantage",Academy of Management Journal, 46(6): 740751.

Cooper, C. (2006):"Knowledgemanagement and tourism",Annals of TourismResearch, 33(1): 4764.

Dyer, J. H. y Hatch, N. W. (2006):"Relation-specificcapabilities and barriers to knowledgetransfers: Creatingadvantagethroughnetworkrelationship",Strategic Management Journal, 27(8): 701-719.

Dyer, J. H. y Singh, H. I. (1998)"Therelationalview: Cooperativestrategies and sources of interorganizationalcompetitiveadvantage",Academy of Management Review, 23(4): 660-679. 
Grant, $\quad$ R. $\quad$ M. (1997):"Theknowledge-basedview of

of thefirm: Implicationsformanagementpractice",Long RangePlanning, 30(3): 450-454.

Grissemann, U., Plank, A. y Brunner-Sperdin, A. (2013):"Enhancingbusiness performance of hotels: The role of innovation and customerorientation",International journal of Hospitality Management, 33: 347-356.

Gulati, R. (1999):"Network location and learning: Theinfluence of networkresources and firmcapabilitiesonallianceformation",Strategic Management Journal, 20(5): 397-420.

Gulati, R., Nohria, N. y Zaheer A. (2000):"Strategicnetworks",Strategic Management Journal, 21(3): 203-215.

Hansen, M. T. (2002):"knowledge network: Explaining effective knowledge sharing in multiunit companies",Organization Science, 13(3): 232-248.

Hjalager, A-M. (2002):"Repairing innovation defectiveness in tourism",Tourism management, 23(5): 465-474.

Hsu, I. C., y Sabherwal, R. (2012):"Relationshipbetweenintellectual capital and knowledgemanagement: Anempiricalinvestigation",DecisionSciences, 43(3): 489-524.

Inkpen, A. C. y Tsang, E. W. (2005):"Social capital, networks, and knowledge transfer",The Academyof Management Review 30(1): 146-165.

Jian, Z. y Wang, C. (2013): "The impacts of networks competence, knowledge, sharing on service innovation performance: Moderating role of relationship quality",Journal of Industrial Engineering and Management, 6(1): 25-49.

Jiménez-Jiménez, D. y Sanz-Valle, R. (2010):"Innovation, organizationallearning, and performance",Journal of Business Research, 64(4): 408-417.

Kang, S. C, Morris, S. S. y Snell, S. (2007):"Relationalarchetypes, organizationallearning and valuecreation: Extendingthe human resourcearchitecture",Academy of Management Review, 32(1): 236-256.

Kim, B. Y. y Oh, H. (2004):"How do hotel firmsobtain a competitiveadvantage?'International Journal of ContemporaryHospitality Management, 16(1): 65-71.

Kogut, B. (2000):"Thenetwork as knowledge: Generative rules and theemergence of structure",Strategic Management Journal, 21(3): 405-425.

Kumar, U., Kumar, V. y de Grosbois, D. (2008):"Development of technologicalcapabilityby Cuban hospitalityorganizations",International Journal of Hospitality Management, 27(1): 12-22.

Laursen, K. y Salter, A. (2006): "Open forinnovation: The role of openness in explaininginnovation performance among U.K. manufacturingfirms",Strategic Management Journal, 27(2): 131150.

Lee, C., Lee, K. y Pennings, J. M. (2001):"Internal capabilities, external networks, and performance: a study on technology-based ventures",Strategic Management Journal 22(6/7): 615- 640.

Leenders,R.Th .A. J., Van Engelen, J. M. L., Kratzer, J. (2003):"Virtuality, communication, and new product team creativity: a social network perspective",Journal of Engineering and technology management, 20(1/2): 69-92.

Leiponen, A. y Helfat, C. E. (2010):"Innovationobjectives, knowledgesources, and thebenefits of breadth",Strategic Management Journal, 31(2): 224-236.

Liu, C-H (2013):"Theprocesses of social capital and employeecreativity: empiricalevidencefromintraorganizationalnetworks",The International Journal of Human Resource Management, 24(20): 3886-3902. 
Martinez-Ros, E. y Orfila-Sintes, F. (2009): Innovationactivity in the hotel industry",Technovation, 29(9): 632-641.

McEvily, B. y Zaheer, A. (1999):"Bridgingties: A source of firmheterogeneity in competitivecapabilities",Strategic Management Journal, 20(12): 1133-1156.

Miller, D. J., Fern, M. J. y Cardinal, L. B. (2007):"The use of knowledgefortechnologicalinnovationwithindiversifiedfirms",Academy of Management Journal, 50(2), 380-326.

Miotti, L. y Sachwald, F. (2003):"Co-operative R\&D: Why and withwhom? Anintegratedframework of analysis",ResearchPolicy,32(8): 1481-1499. internalrelationshipsinfluencingcustomerrelationshipquality and company performance",Journal of Business \& Industrial Marketing, 27(6): 486-496.

Molina J. (1999):"Dialogue: Ontherelationalview",Academy of Management Review, 24(2): 184186.

Molina-Morales X. y Martínez-Fernández, M. T. (2009):"Toomuchlove in theneighborhood can hurt: Howanexcess of intensity and trust in relationshipsmay produce negativeeffectsonfirms",Strategic Management Journal, 30(9): 1023-1023.

Mowery, D. C., Oxley, J.E. y Silverman, B. S. (1996): "Strategicalliances and interfirmknowledge transfer",Strategic Management Journal, 17(S2): 77-91.

Nahapiet, J. y Ghoshal, S. (1998):"Social capital, intellectual capital, and theorganizationaladvantage",Academy of Management Review, 23(2): 242-266.

Nonaka, I. y Takeuchi, H. (1995):Theknowledge-creatingcompany,New York, Oxford UniversityPress.

Novelli, M., Schmitz, B. y Spencer, T. (2006): "Networks, clusters and innovation in tourism: A UK experience",Tourism Management, 27(6): 1141-1152.

Ooi, C-A., Hooy, C-W.ySom, A. P. M. (2014): "Diversity in human and social capital: Empirical evidence from Asian tourism firms in corporate board composition",Tourism Management 48: 139-153.

Ordanini, A. y Maglio, P. P. (2009):"Marketorientation, internalprocess, and externalnetwork: A qualitativecomparativeanalysis of key decisional alternatives in the new servicedevelopment",DecisionSciences, 4O(3): 601-625.

Ordanini, A. y Parasuraman, A. (2011):"Serviceinnovationviewedthrough a servicedominantlogiclens: A conceptual framework and empiricalanalysis",Journal of ServiceResearch, 14(1): 3-23.

Pavlovich, K. (2003):"The evolution and transformation of a tourism destination network: the Waitomo Caves, New Zealand",Tourism Management, 24(2): 203-216.

Perry-Smith, J. E. (2006):"Social yetcreative: the role of social relationships in facilitating individual creativity",Academy Management Journal, 49(1): 85-101.

Pikkemaat, B. y Peters, M. (2005):"Towardsthemeasurement of innovation-A pilotstudy in thesmall and mediumsized hotel industry",Journal of QualityAssurance in Hospitality\&Tourism, 6(3): 89-112.

Powell, W. W., Koput, K. W. y Smith-Doerr, L. (1996):"Interorganizationalcollaboration and the locus of innovation: Networks of learning in biotechnology",AdministrativeScienceQuarterly, 41(1): 116-145.

Quintana-García, C y Benavides-Velasco, C. A. (2007): "Concentraciones territoriales, alianzas estratégicas e innovación. Un enfoque de capacidades dinámicas",Cuadernos de Economía y Dirección de Empresa, 30: 5-38. 
Rhee, J. Park, T. y Lee, D. H. (2010): "Drivers of innovativeness and performance forinnovativeSMEs in South Korea: Mediation of learningorientation",Technovation, 30(1): 6575.

Sarkar, M., Echambadi, R. y Harrison, J. S. (2001): "Alliance entrepreneurship and firm market performance",Strategic Management Journal, 22(6/7): 701-711.

Saxena, G. (2005):"Relationships, networks and thelearningregions: Case evidencefromthePeakDistrictNational Park",Tourism Management, 26(2): 277-289.

Scott, N., Cooper, C. y Baggio, R. (2008): "Destination networks four Australian cases",Annals of TourismResearch, 35(1): 169-188.

Scott, N. y Laws, E. (2006): "Knowledge Sharing in Tourism and Hospitality",Journal of Quality Assurance in Hospitality \& Tourism, 7(1): 1-12.

Sorensen, F. (2007):"Thegeographies of social networks and innovation in tourism",TourismGeographies, 9(1): 22-48.

Starbuck, W. H. (1992): "Learning by knowledge-intensive firms",Journal of Management Studies, 29(6): 713- 740.

Subramaniam, M. y Youndt, M. A. (2005): "The influence of intellectual capital on the types of innovative capabilities",Academy of Management Journal, 48(3): 450-463.

Tinsley, R. y Lynch, P. (2001):"Small tourism business networks and destination development",International Journal of Hospitality Management, 20(4): 367-378.

Tajeddini, K. (2010):"Effect of customerorientation and entrepreneurialorientationoninnovativeness: Evidencefromthe hotel industry in Switzerland",Tourism Management, 31(2): 221-231.

Tajeddini, K. y Trueman, M. (2012):"ManagingSwissHospitality: How cultural antecedents of innovation and customer-orientedvaluesystems can influence performance in the hotel industry",International Journal of Hospitality Management, 31(4): 1119-1129.

Tsai, K.H. (2009): "Collaborativenetworks and productinnovation performance: Toward a contingencyperspective", ResearchPolice, 38(5): 765-778.

Tsai, W. (2001):"Knowledge transfer in intra organizational networks: Effects of network position and absorptive capacity on business unit innovation and performance", Academy of Management Journal, 4(5): 996-1004.

Van de Ven, A. H. (1986): "Central problems in the management of innovation", Management Science, 32(5): 590-607.

Wang, T. y Bansal, P. (2012): "Social responsibility in new ventures: Profiting from a long-term orientation",Strategic Management Journal, 33(10): 1135-1153.

Wang, Y. y Fesenmaier, D. R. (2007): "Collaborative destination marketing: A case study of Elkhart county, Indiana",Tourism Management, 28(3): 863--875.

Weiermair, K. (2006):"Product improvement or innovation: Whatis the key to success in tourism? "OECD Conference Papers: Innovation and Growth in Tourism, Lugano, Switzerland, 18-19 September 2003. Acceso 2015, 06, disponible en: http://www.oecd.org/cfe/tourism/34267947.pdf

Young, G. J., Charns, M. P. y Shortell, S. M. (2001):"Top manager and network effects on the adoption of innovative management practices: a study of TQM in a public hospital system", Strategic Management Journal, 22(10): 935-951. 
Rivero Almeida, L. y Nieves Rodríguez, L. (2016): "Relaciones sociales y desempeño organizativo: Estudio empírico en empresas del sector hotelero "

\section{HOW TO CITE THIS ARTICLE IN BIBLIOGRAPHIE}

Rivero Almeida, L. y Nieves Rodríguez, L. (2016): "Relaciones sociales y desempeño organizativo: Estudio empírico en empresas del sector hotelero" Rotur. Revista de Ocio y Turismo, 11: 77-94, ISSN-e 2695-6357 DOI: https://doi.org/10.17979/rotur.2016.11.1.1782 